Review Article

\title{
Status of Neuropharmacology Research in India
}

\author{
YOGENDRA K GUPTA*, JATINDER KATYAL and SANGEETHA GUPTA \\ Neuropharmacology Laboratory, Department of Pharmacology, All India Institute of Medical Sciences, \\ New Delhi 110 029, India
}

(Received on 13 September 2017; Accepted on 11 December 2017)

\begin{abstract}
Neurological and neuropsychiatric problems have been recognized as formidable issue worldwide in developed as well as developing countries due to a sizable mortality and debilitating sequelae. A lot of impetus on research in this area over the past two decades has led to a better understanding of these diseases and made available tools in the form of experimental model systems, mechanistic pathways and genetic connections to provide the researchers with an enabling environment for drug discovery, better value from existing drugs and circumventing toxic effects. In India, the past decade has witnessed a broadening of research base of neuropharmacology research and a close look reveals that Indian research is very much contemporary. A kaleidoscopic view of neuropharmacology research in India in over the past five years is presented in this article.
\end{abstract}

Keywords: Neuropharmacology; Research; Neurological Disorders; Neuropsychiatric Disorders; Epilepsy; Stroke; Parkinson's Disease; Alzheimer's Disease; Pain

\section{Introduction}

Neuropharmacology is a branch of pharmacology, which evolved with only four drugs prior 1900s namely morphine and aspirin for pain, nitrous oxide as anesthetic agent for surgery and caffeine for drowsiness. Neurological disorders (NDs) in a nutshell are the one that impairs the brain, spinal cord, neuromuscular function or peripheral nerve by affecting central nervous system (CNS) or the peripheral nervous system. According to World Health Organization (WHO), NDs have been categorized into two major categories for disability-life adjusted years (DALYs) and death data as: (i) the neuropsychiatric category including epilepsy, Alzheimer and other dementias, Parkinson's disease, multiple sclerosis and migraine. (ii) NDs from other categories include diseases and injuries which have neurological sequelae include cerebrovascular diseases, poliomyelitis, tetanus, meningitis and Japanese encephalitis. NDs contributing almost $6 \%$ of global burden of disease and $12 \%$ of total deaths worldwide have been a major public health challenge. Within these, more than half of the burden in DALYs is constituted by cerebrovascular diseases which are responsible for $85 \%$ of the deaths, $12 \%$ by Alzheimer and other dementias and $8 \%$ each by epilepsy and migraine. The lower middle income countries account $16.8 \%$ of the total deaths as compared to $13.2 \%$ in high income countries (WHO 2006).

In 1960s, epidemiological studies were restricted to only stroke and epilepsy, but now various programs such as Neuroscience program of WHO are promoting focus on other NDs also (Gourie-Devi, 2014). In India the crude prevalence rate varies from 967-4,070 per 100000 population accounting approximately 30 million people suffering from NDs among 1.27 billion population, of which approximately 6 to 8 million people are suffering from epilepsy, 9.4 million deaths with $20-30 \%$ of strokes occurring in people younger than 45 years of age and crude prevalence rate for Parkinson's disease was reported to be 6-53/100000 (Gourie-Devi, 2014).

Currently available medications are with inadequate efficacy, limitations of time-bound administration, associated with significant side-effects 
and not affordable for most patients in developing countries. Hence, there is need for research into treatment options for these disorders. The highlights of research in neuropharmacology along with various labs, which are actively working in India, are discussed.

\section{Experimental and Clinical Studies in Stroke}

Stroke or cerebrovascular disease refers to an umbrella of conditions caused by an interruption of blood supply to brain either due to bursting of blood vessels or blockage due to a clot, leading to brain tissue damage due to an insufficient supply of oxygen and nutrients. The consequences include motor, sensory, visual and neuropsychological symptom deficits. Stroke ranks fifth as cause of death after heart, cancer and chronic lower respiratory disease along with unintentional injuries/accidents, worldwide. Twenty percent of patients having their first stroke die within a month and of those alive till six months, about a third are dependent on others. The number of people affected annually is over 2 million worldwide, is estimated to increase dramatically in the coming years and to increase by one half by year 2020 .

In a recent survey in India stroke accounted $13 \%$ of all mortality cases after coronary artery disease (Banerjee and Das, 2016). According to an Indian Council of Medical Research (ICMR) survey, stroke stands out as the second commonest cause of death in India. There are 1,65,000 stroke cases each year, approximately one stroke every 40 seconds and one death every 4 minutes (Padma, 2016). Analysis of community surveys from different regions of India shows a crude stroke prevalence rate of about 90 222 cases per 100,000 populations above 20 years of age, amounting to a total of about 1 million deaths. The male to female ratio is 1.7 , accounting $1.2 \%$ of total deaths. Significant risk factors are hypertension accounting $57 \%$ of all stroke deaths and diabetes mellitus (Gourie-Devi, 2014). From global data of 2004, the stroke-related DALY loss in India was 597.6 per 100,000 person-years (Banerjee and Das, 2016).

In India in the last ten years, stroke research preclinical as well as clinical has grown with many labs actively involved in research in stroke, under the guidance of eminent researchers. At the All India Institute of Medical Sciences (AIIMS), New Delhi, the neuropharmacology lab, Department of
Pharmacology has carried out some pioneering work in this area. The experimental model standardized to induce focal cerebral ischemia i.e. intraluminal filament middle cerebral artery occlusion for $2 \mathrm{~h}$ in rats has been used to unravel the mechanistic pathways and design novel therapies ranging from phytomedicines, immunosuppressant's rapamycin and mycophenolate mofetil, vineatrol, combination treatment of TAK-044, an endothelin antagonist and aspirin, an anti-inflammatory agent, lercanidipine a calcium channel blocker with neuroprotective effect mediated through anti-oxidant, anti-inflammatory, vasodilatory and anti-apoptotic property (Gupta et al., 2017a; 2017b; Chauhan et al., 2012; Briyal et al., 2007). New dimensions are constantly being added and training has been imparted to scores of researchers and students all over the country. The protocol for assessment now is not restricted to behavioural, biochemical and histopathological but monitoring of cerebral blood flow (CBF) by Laser Doppler flowmetry in the lab alongwith magnetic resonance imaging (MRI) and proton nuclear magnetic resonance spectroscopy $\left({ }^{1} \mathrm{H} \mathrm{NMR}\right)$ is being routinely employed in collaboration with the Department of NMR \& MRI Facility, AIIMS, New Delhi.

Esters of fumaric acid are the activators of nuclear factor, erythroid 2 like pathways, leading to transcription of a number of neuroprotective genes like heme oxygenase-1, NAD $(\mathrm{P}) \mathrm{H}$ dehydrogenase (quinone)-1 etc. These are being evaluated in stroke. AIIMS has also taken a lead in clinical studies such as Family-led Rehabilitation after Stroke in India (ATTEND) trial, first time introduction of telestroke model in India and applications of stem cell therapy (ATTEND Collaborative group, 2017; Sharma et al., 2016; Devi et al., 2016).

National Institute of Pharmaceutical Education and Research (NIPER)-Mohali have established both MCAo model of focal cerebral ischemia and bilateral carotid artery occlusion (BCCAO) model of global cerebral ischemia. The neuroprotective potential of the newer thyrotropin-releasing hormone analogue LpGlu-(1-benzyl)-1-His-1-Pro- $\mathrm{NH}_{2}$ (Rajput et al., 2016), a potent and selective neuronal nitric oxide synthase inhibitor, 3-bromo-7-nitroindazole and sodium 4-phenylbutyrate protected against endoplasmic reticulum stress and focal cerebral ischemicreperfuison injury associated with comorbid type 2 
diabetes has been evaluated (Srinivasan and Sharma, 2012a; 2012b). Other contributions included hepatocyte nuclear factor-1alpha mediated albumin over expression in ischemic rat brain, evaluation of neuroprotective agents such as trolox, pioglitazone, pifthrin-alpha and peroxynitrite scavengers (MnTMPyP and FeTPPs) (Prajapati et al., 2012).

NIPER-Ahmedabad is targeting stroke by intraarterial stem cells. Studies performed suggested that intra-arterial mesenchymal stem cells (MSCs) therapy was promising treatment for stroke (Jadhav et al., 2015). Since in stroke, MSCs mediated neuroprotection occurs via brain-derived neurotrophic factor (BDNF), development of drug delivery system that can transport BDNF across blood brain barrier is being explored (Sarmah et al., 2017; Atchaneeyasakul et al., 2016) as well as for clinical trials.

\section{Experimental and Clinical Studies in Epilepsy}

Epilepsy is a neurological disorder with an overall prevalence of 50 million people worldwide and approximately 6-10 million people in India (Megiddo et al., 2016). As per Western data, the morbidity and mortality due to epilepsy is second only to stroke. Devinsky et al. (2016). In a recent review (Devinsky et al., 2016) it has been pointed out that epilepsyrelated mortality accounts for substantial premature mortality as the incidence of epilepsy is greatest among young , remains under appreciated and public health interventions are inadequate. Despite the major advances in antiepileptic drug (AED) therapy, about one third of patients with epilepsy still do not have adequate seizure control with currently available AEDs when prescribed as monotherapy, leading to use of two or more AEDs concomitantly and associated problems like drug toxicity and drug-drug interactions.

In the Department of Pharmacology, AIIMS, New Delhi, multiple experimental seizure models have been standardized and are routinely employed. These include not only the models for screening antiseizure activity of compounds like pentylenetetrazole (PTZ)induced seizures, maximal electroshock induced seizures and chemical kindling but also models mimicking special situations viz., antiepileptic drug (AED) tolerance, refractory intractable seizures, excitotoxicity, post-traumatic seizures and epileptogenesis.
While, efforts are on to elucidate basic mechanisms to look for newer targets, validating phytomedicines in seizures models and also in combination with antiepileptic drugs remains a major thrust area. In this series, the interaction profile of hydroalcholic extract of Zizyphus jujuba (HEZJ), Acorus calamus, Curcuma longa, Ocimum sanctum with AEDs like phenytoin, sodium valproate, and carbamazepine has been explored in experimental seizure models, behavioural studies and using biochemical paradigms with encouraging results. To rule out a pharmacokinetic interaction serum levels of AEDs using high-pressure liquid chromatography (HPLC) are routinely measured (Pahuja et al., 2012a; Pahuja et al., 2013; Pahuja et al., 2012b; Katyal et al., 2012; Sarangi et al., 2017).

Validation studies for other complementary and alternative systems of medicines is also underway and in this series, co-administration of Panchagavya Ghrita, a cow product composed of mainly five components; cow milk, clarified butter from cow milk, cow urine, curd from cow milk, and cow dung juice, with low doses of phenytoin and carbamazepine showed complete seizure protection in MES and PTZ model of seizures (Joshi et al., 2015a; 2015b). Selective COX-2 inhibitor etoricoxib also reduced seizures, oxidative stress, and learning and memory in pentylenetetrazole PTZ $(30 \mathrm{mg} / \mathrm{kg}$, i.p. ) kindled rats. The anticonvulsant effect was evident at lower doses $(1 \mathrm{mg} / \mathrm{kg})$, which was reversed/reduced at higher dose $(10 \mathrm{mg} / \mathrm{kg})$ (Katyal et al., 2015). Curcumin, a dietary antioxidant was studied against kainic acid (KA, 10 $\mathrm{mg} / \mathrm{kg}$, ip) induced seizures and nutraceutical zinc was found to have protective effect against seizures (Kumar et al., 2015).

A lot of work on mechanisms of actions has also been undertaken. In order to study the involvement of N-methyl-D-aspartate (NMDA) and opioid receptors, MK801, an NMDA antagonist and naloxone, an opioid receptor antagonist, were administered with tianeptine. The anticonvulsant effect of tianeptine was potentiated upon combination with MK801, while it was mitigated with naloxone (Reeta et al., 2016).

Besides evaluating potential stand alone and add on therapies in experimental models, lot of attention is now being focused on co-morbid conditions like 
cognitive impairment and depression in persons with epilepsy (PWE) and other perplexing problems like sudden unexpected death in epilepsy (SUDEP), AED tolerance and drug resistant or refractory seizures, neurogenesis and angiogenesis inhibitors and epileptogenesis.

On the clinical side, till date there is a paucity of proper epidemiological and clinical studies on different aspects of epilepsy in India. This is now being addressed by many clinicians and basic scientists across the country. At AIIMS, in an observational study in PWE, adverse events and drug load (prescribed daily dose/defined daily doses) of AEDs were evaluated. There were higher AEs and lower seizure control in PWE treated with polytherapy in combination of greater than or equal to three AEDs as compared to both monotherapy and combination of two AEDs (Joshi et al., 2017). In another crosssectional study the body composition and lipid profile among PWE on newer and conventional AEDs was compared. It was concluded that treatment responses are affected in patients with existing altered body composition with valproic acid as compared with other AEDs like levetiracetam, carbamazepine and phenytoin (Sarangi et al., 2016). In yet another crosssectional study, as phenytoin is mainly metabolized by cytochrome $\mathrm{P} 450$ enzyme, the prevalence of cytochrome P450 CYP2C9 polymorphisms in Indian epileptic children was investigated alongwith monitoring phenytoin serum levels. The heterozygous polymorphism was observed which was more common in CYP2C9*3 than *2 along with elevated serum phenytoin levels (Chaudhary et al., 2016). Similarly, uridine 5'-diphospho (UDP)glucuronosyltransferase (UGT) which metabolizes around $50 \%$ of valproate, frequencies of UGT1A6 geneotypes and alleles were reported in another cross-sectional study (Jain et al., 2015). A strong correlation was observed between serum and saliva levels with valproate and with mono-therapy or bitherapy, however, no significant association was observed with poly-therapy in PWE (Dwivedi et al.,2015; 2016). Subclinical hypothyroidism with longterm valproate therapy and elevated blood ammonia levels at higher dose, $40-60 \mathrm{mg} / \mathrm{kg} / \mathrm{d}$ was observed in epileptic children (Sahu et al., 2012). In another crosssectional study no significant differences in the levels of trace elements was observed with conventional as compared to newer AEDs treatment in PWE (Sarangi et al., 2014).

Another institute actively involved in epilepsy research is School of Pharmaceutical Education and Research (SPER), Jamia Hamdard, New Delhi. Most of the research work is focused on investigating the novel molecular targets for epilepsy and on epilepsy associated complications. Some of the highlights include evaluation of histamine $\mathrm{H}_{3}$ receptor inverse agonism and glycogen synthase kinase- $3 \beta$ inhibition, aromatase inhibition, selective estrogen receptor modulation in hippocampus for postmenopausal temporal lobe epilepsy (TLE), $\mathrm{N}$-acetylcysteine and medroxyprogesterone, sodium hydrogen exchange inhibitors as novel targets for epilepsy associated neurotoxicity especially for TLE, the most drugresistant form of epilepsy (Bhowmik et al., 2014; 2015; Devi et al., 2012; Nasir et al., 2012; Pottoo et al., 2014; Rashid et al., 2015).

Other lines of research are AEDs-associated osteoporosis and obsessive compulsive symptoms in patients with drug-resistant TLE and the involvement of serotonergic system dysregulation in obsessive compulsive disorder (OCD). A case report of carbamazepine in alleviating symptoms of OCD, the effect of oxcarbazepine, a second generation AED, on first ever mouse models of OCD was investigated (Anwar et al., 2014; Arora et al., 2013).

Some of the projects in Postgraduate Institute of Medical Education and Research (PGIMER), Chandigarh have shown strong evidence that inflammation can cause increased seizure susceptibility. Towards management of epilepsy, pharmacokinetic interaction of different drugs with phenytoin and carbamazepine, artemisnin compound, etoricoxib, aceclofenac, newer proton pump inhibitors etc. have been evaluated. Therapeutic drug monitoring (TDM) pattern of antiepileptic drugs and lithium were also analyzed which will be of help to other centers offering TDM service for hospital patients (Kumar et al., 2017a; Sewal et al., 2017)

At Vallabhai Patel Chest Institute (VPCI), Delhi, it has been demonstrated that anxiety and seizures are closely similar neurobehavioural disorders and are regulated by limbic system. Gulati et al., (2014) evaluated the regulatory roles of NO in these pathophysiological states in experimental models. In test for anxiety, aminophylline induced anxiogenic 
responses, which was accompanied by increases in MDA levels and reductions in GSH and NOS activity in brain homogenates - changes which were reversed by L-arginine and melatonin pretreatments.

\section{Alzheimer's Disease}

Alzheimer's disease (AD) is a progressive neurological condition in elderly, which continues to be a major challenge for neuroscientists. The hallmarks of disease (amyloid $\beta$ plaques and neurofibrilliary tangles formation) are associated with elevated levels of advanced glycation end products, oxidative damage and neuro-inflammation. In addition to classical cholinergic dysfunction which is primarily responsible for diminished cognitive functions, multiple neurotransmitter systems are deranged in AD. Genetic and environmental factors and the gene-environment interaction make the response to commonly used drugs such as donepezil, rivastigmine, galantamine and memantine less predictable and often unsatisfactory.

CSIR-Central Drug Research Institute (CDRI), Lucknow has made significant contribution to the neuropharmacology of several CNS diseases, including AD. A series of research articles by Nath and colleagues clearly demonstrated that okadaic acid (OKA), a polyether toxin which acts as a potent inhibitor of protein phosphatase PP1 and PP2A, induces hyper phosphorylation of tau, a hallmark feature of AD (Kamat et al., 2013; 2014; Kamat and Nath, 2015). Besides this pharmacological model, streptozotocin (STZ)-induced experimental model of dementia/AD had also been extensively used to delineate various pathways as well as effect of several phytochemicals from CDRI. It was shown that STZ induced neuronal death involved modulation of mitochondrial functioning, endoplasmic reticulum stress and DNA fragmentation (Biswas et al., 2016; 2017) and that the activation of glial cells and post synaptic neurotoxicity is one of the key event in memory impairment (Rai et al., 2014). With regard to the effect of phytochemicals in $\mathrm{AD}$, standardized extract of Bacopa monniera was shown to attenuate the memory dysfunction in rats (Dwivedi et al., 2013), anti-inflammatory and anti-proliferative effects of guggulipid was also reported by CDRI (Niranjan et al., 2014). The investigation on clinically used nootropic drug piracetam at CDRI revealed that it could offer neuroprotection through mitochondria mediated caspase-independent pathway and also has antioxidative and anti-apoptotic activity (Verma et al., 2015). These observations related to mechanism of action of piracetam potentially can stimulate wide clinical utilization in future. Another interesting study has been done by (Rai et al., 2014) which showed that insulin offers a protective effect in STZ induced toxicity in rats.

CSIR-CDRI, Lucknow, added to its fold a new facility that utilizes powerful genetics of model system C. elegans towards understanding mechanistic aspects of age associated neurodegenerative diseases. The transgenic strains of $C$. elegans that are employed, express human amyloid beta (for AD) and human alpha synuclein for Parkinson disease (PD). The model is very precious as it has been shown to exhibit similar proteomic expression pattern as that of post-mortem human diseased brain. The model has been established for studying protein aggregation, effect on mitochondrial content, effect on reactive oxygen species and for studying effect on excitatory neurotransmission, thus providing with a whole organismal environment towards studying the multifactorial aspect of these diseases. The model presents with easy to score endpoints, making it suitable for rapid initial screening. A repository of 60 transgenic and mutant $C$. elegans strains that could be employed for studying various biological processes relevant to human diseases is being maintained. Studies with the model have helped identify novel modulators of AD and PD associated endpoints. The laboratory has carried out systematic functional genomics screens towards identifying modulators like ceIDE-1 (Haqueand Nazir, 2016a), sma-9 (Haque and Nazir, 2016b), cat-2 (Jadiya et al., 2012), ida-1 (Fatima et al., 2014) and micro-RNA molecules like Let-7in the context of neurodegenerative diseases. Further, methods for efficient RNAi induced silencing, that can work even in transgenic/mutant backgrounds, have been established by the laboratory (Jadiyaand Nazir, 2014). Going forward, this group is working on small RNA molecules including circular RNA and micro RNA towards possibly establishing theronostic tools that can be exploited for therapy as well as early detection of neurodegenerative diseases.

In the Department of Pharmacology, AIIMS, New Delhi, intracerebroventricular (ICV) STZinduced model of Alzheimer like conditions is 
established and numerous potential neuroprotective agents screened.

Since, in Ayurvedic system of medicine, several plants have been used in memory related disorders. In a series of experimental studies, the effect of hydroalcoholic and aqueous extracts of Clitoria ternatea and Evolvulus alsinoides medicinal plants were investigated in ICV-STZ induced model of Alzheimer like conditions in rats. Various in-vitro screening methods as complementary parameters have been standardized for following activities: oxygen radical antioxidant activity, 2,2'-azino-bis(3ethylbenzothiazoline-6-sulphonic acid (ABTS) free radical scavenging activity, peroxynitrite radical antioxidant activity, acetycholinesterase (AChE) andpseudocholinesterase (BuChE), prolyl endopeptidase, rho kinase (ROCK II) and GSK-3 3 . Memory improvement is assessed using a battery of neurobehavioural parameters (Morris water maze, passive avoidance, elevated plus maze and photoactometer) on $14^{\text {th }}, 21^{\text {st }}$ and $28^{\text {th }}$ day after STZ injection. Hydro-alcoholic extract as compared to aqueous extract of Clitoria ternatea and Evolvulus alsinoides was found to be superior and dose dependently (100,300 and $500 \mathrm{mg} / \mathrm{kg}$, p.o.) prevented STZ induced cognitive impairment via reduction of oxidative stress and rho kinase expression and improved cholinergic dysfunction (Mehla et al., 2012; 2013a; 2013b).

Similarly, taurine, an essential amino acid and edaravone, a potent free radical scavenger were investigated in ICV-STZ model of cognitive impairment in male Wistar rats. (Reeta et al., 2017a; 2017b). Currently, magnetic stimulation is being evaluated in $\mathrm{AD}$ in collaboration with physiology department

Jamia Hamdard, New Delhi has been actively working on the role of MAPK-activated protein kinase 2 in neuro inflammation and insulin signalling pathway in neurons during $\mathrm{AD}$ and to identify molecular targets that are found to be affected most and could be used as a therapeutic target against neurodegeneration in $\mathrm{AD}$. Insulin resistance has been demonstrated to be responsible for neurodegeneration in various in vitro, in vivo and even in post-mortem analysis of brain of patients suffering from AD (Singh et al., 2017a; 2017b). The role of hypercholestero- lemia in neuroinflammation and memory function along with markers of oxidative stress and cholinergic activity in brain were also evaluated. Rosuvastatin ameliorated cognitive impairment in female Wistar rats fed with high-salt and cholesterol diet (HSCD) by acting as a dual inhibitor of AChE and amyloid-beta $\left(\mathrm{A} \beta_{1-42}\right)$ peptide aggregation (Husain et al., 2017a). Rosuvastatin also modulated neuronal cell death by inhibiting the overexpression of nuclear factor kappaB (NF-kB) and production of nitric oxide (NO), tumor necrosis factor alpha and interleukin-10 against HSCD model (Husain et al., 2017b). Spatial memory impairment reduced $\mathrm{CBF}$ and acetylcholine levels were evident after ICV angiotensin II, which was mediated through AT1 receptors (Tota et al., 2013a; 2013b). Scopolamine-induced memory impairment mediated through central AChE and CBF was established, which was reversed by perindopril treatment in mice (Tota et al., 2012a). OKA is a potent inhibitor of protein phosphatases 1/2A (PP2A) leading to hyperphosphorylation of Tau protein a characteristic feature of AD. NMDA antagonists MK801 and memantine attenuated OKA-induced neurotoxicity thereby implicated role of NMDA receptors in OKA (ICV)-induced tau hyperphosphory lation (Kamat et al., 2013). OKA (200 ng, ICV), apart from memory impairment and mitochondrial dysfunction, induced cholinergic dysfunction and neuronal loss, which was reversed by antidementia drugs like donepezil and memantine (Kamat et $a l ., 2012)$. Studies on the role of central reninangiotensin system in memory and its interaction with BDNF in ICV-STZ have been established (Tota et al., 2012b).

University Institute of Pharmaceutical Sciences (UIPS), UGC Centre of AdvancedStudies (UGCCAS), Panjab University, Chandigarh, is working on neurodegenerative including age related diseases and related complications, particularly cognitive dysfunctions. Their primary focus is on common mechanisms such as oxidative stress, mitochondrial dysfunction, glutamatergic and neuroinflammatory. Stroke-induced memory dysfunction involving glutathione was studied. BCCAO-induced memory dysfunction was ameliorated by celecoxib (a selective cyclooxygenase-2 [COX-2] inhibitor) and nimesulide (a preferential COX-2 inhibitor) (Gaur and Kumar, 2012). 
In PGIMER, Chandigarh, effect of mesenchymal stem cells and galantamine nanoparticles; granulocyte colony stimulating factor alone and in combination with memantine in rat model of AD has been shown to be protective (Misra et al., 2016; Prakash et al., 2013a; 2013b).

The effects of NO modulators in ICV-STZ, 3 $\mathrm{mg} / \mathrm{kg}$ induced rat model of sporadic AD (sAD) was evaluated in VPCI, Delhi. Dubey and co-workers inferred that $\mathrm{NO}$ and its interactions with reactive oxygen species could reduce age related cognitive deficits and that L-arginine could be a potential therapeutic supplement in the treatment of sAD (Dubey et al., 2017a; 2017b).

\section{Depression, Psychosis, Anxiety, Post-traumatic Stress Disorders}

Two labs in CSIR-CDRI, Lucknow are working on psychiatric disorders-mainly psychosis and depression. Palit and colleagues have evaluated effectiveness of several phytochemicals such as Panax quinquefolium(), Bacopa monnieri (Chatterjee et al., 2012; 2015). Palit and colleagues have also shown salutary effect of several phytochemicals and plant extracts on stress induced anxiety and depression. Thus, Plumeria rubra exhibited anxiolytic effects in mice (Chatterjee et al., 2013) and novel Ocimumoside $\mathrm{A}$ and $\mathrm{B}$ exhibited antidepressant effects in chronic unpredictable stress model in rats (Ahmad et al., 2012). In a recent and quite elegant study, Yadav and colleagues revealed that sustained activity of kappa opioid receptor, upregulated NR2B-NMDA activity in hippocampus and acts as molecular determinants of treatment resistant depression in mice. This observation for the first time demonstrated the essential relationship between two clinically very relevant pathways that are being pursued for the development of better therapeutics for depression (Dogra et al., 2016).

Agomelatine, a novel antidepressant with agonistic actions at melatonergic (MT1 and MT2 receptors) and antagonistic actions at 5HT-2C receptors augmented the antidepressant effect of venlafaxine independent ofkynurenine pathway as evidenced by failure to reduce hippocampal indole amine 2, 3 dioxygenase activity (Thomas et al., 2016).

At Banaras Hindu University, Varanasi, modulation of hypothalamic-pituitary-adrenal axis and brain monoaminergic systems in a rat model of stress by eugenol is being explored. Further eugenol was evaluated against restraint stress-induced gastrointestinal dysfunction (Garabaduet al., 2015). Shilajit attenuated behavioural symptoms of chronic fatigue syndrome by modulating the hypothalamicpituitary-adrenal axis and mitochondrial bioenergetics in rats (Surapaneni et al., 2012). Asparagus racemosus modulated the hypothalamic-pituitaryadrenal axis and brain monoaminergic systems in rats (Krishnamurthy et al., 2013a). Asparagus racemosus also illustrated antidepressant activity, attenuated anxiety-like behaviour and enhanced memory against amnesia in rodent models (Garabadu et al., 2014;). Risperidone in ultra low dose protected against stress in the rodent cold restraint model by modulating stress pathways. Furthermore, risperidone gastro protective potential was evaluated against stress and pyloric ligation induced gastric lesions. Citalopram protected against cold-restraint stressinduced activation of BDNF and expression of nuclear factor kappa-light-chain-enhancer of activated B cells in rats (Garabadu et al., 2015). Diazepam potentiated the antistress and anxiolytic activities of metformin in type-2 diabetes mellitus with co-occurring stress in experimental animals (Garabadu et al., 2014). Risperidone was found to ameliorate post-traumatic stress disorder (PTSD)-like symptoms in modified stress re-stress model (Krishnamurthy et al., 2013b). Risperidone attenuated modified stress-re-stress paradigm-induced mitochondrial dysfunction and apoptosis in rats exhibiting PTSD-like symptoms (Garabadu et al., 2015). At Neuropharmacology research laboratory of Department of Pharmaceutics, IIT (BHU), potential neuropsycho-pharmacological activities of analytically characterised extracts edible herb (Brassica juncea) and Ayurvedic herb (Andrographis paniculata) was evaluated in diabetic rodents (Thakur et al., 2013; 2014a; 2014b; 2016). Authors have reported that standardised extract of Andrographis paniculata (AP) and isolated pure compound (andrographolide) showed adaptogenic activities and protective effect in foot-shock induced stressed rodents (Thakur et al., 2014c; 2014d). Authors have warranted that both AP and andrographolide are pharmacologically polyvalent antistress agents, and that biological processes regulating corticosterone and cytokine homeostasis are involved 
in their modes of actions.

Department of Neuropsychopharmacology, Institute of Human Behaviour \& Allied Sciences is providing regular therapeutic drug monitoring (TDM) service in the field of neuropsychiatry for last 20 years with lowest turnaround time. The department is one of the major providers of TDM service in North India. The work on tools for measuring drug use and adverse effects in neuropsychiatry practice and also on prevalence and factors associated with medication compliance in mental disorders will go a long way in providing insights into enabling factors which can improve compliance in this group of patients (Sharma et al., 2017).

\section{Parkinson's and Huntington's Disease}

Besides AD, several interesting and insightful studies with respect to etiology and pathophysiology of PD in preclinical models were also reported form CDRI in recent years. The studies on Rotenone model of Parkinsonism have suggested that both oxidative stress and endoplasmic reticulum stress play significant role in PD pathology (Goswami et al., 2016a; 2016b; Gupta et al., 2015). Further investigation revealed the critical role on inducible nitric oxide synthase in Parkinson's pathology involving mitochondrial impairment and apoptosis (Gupta et al., 2015). A series of studies have shown the therapeutic utility of acetyl-L-carnitine in rodent models of PD by demonstrating the essential role of $\mathrm{Wnt} / \beta$-catenin pathway in glial cells for neurogenesis (Singh et al., 2015; 2017a). This group also demonstrated the beneficial effect of MK801, a non-competitive NMDA antagonist, in Parkinsonian rats via modulation of hippocampal neurogenesis (Singh et al., 2017b).

The results of studies at Banaras Hindu University, Varanasi, include pretreatment with silibinin attenuated biochemical and behavioural changes induced by intrastriatal $\mathrm{MPP}^{+}$injection in rats (Geed et al., 2014). Coenzyme Q10 prevented mitochondrial dysfunction and facilitated pharmacological activity of atorvastatin in 6-OHDA induced dopaminergic toxicity in rats (Prajapati et al., 2017). Purinergic antagonism prevented mitochondrial dysfunction and behavioural deficits associated with dopaminergic toxicity induced by 6-OHDA in rats (Kumar et al., 2017b).
National Institute of Mental Health and Neurosciences (NIMHANS), Bangalore has explored the role of redox and mitochondrial dynamics in neurodegenerative and myodegenerative conditions. The neuroprotective effects of Bacopa monnieri and curcuminin models of PD (Shinomol et al., 2012a) and Huntington's disease (HD) (Shinomol et al., 2012b) were evaluated. In NIPER-Mohali beneficial effects of pioglitazone, a peroxisome proliferatoractivated receptor (PPAR)- $\gamma$ agonist on cognitive impairment in 1-methyl-4-phenyl-1,2,3,6tetrahydropyridine (MPTP) model of PD. In addition neuroprotective potential of PPAR- $\beta / \delta$ agonist and PPAR- $\alpha$ agonist in cognitive impairment in PD model were also demonstrated (Das et al., 2014a; Uppalapati et al., 2014).

\section{Sleep Disorders and Autism Spectrum Disorders}

Research work from, NIMHANS has focused mainly into the neurophysiological changes such as fear memory, extinction, sleep architecture and neural processing in the limbic circuit. They found early maternal separation stress, irrespective of timing and duration, resulted in abnormal sleep architecture accompanied with the abnormal neural processing between limbic circuit during rapid eye movement sleep (REM) (Sampath et al., 2014). In addition, the study also found abnormal increase in anxiety, fear memory retention and impaired fear extinction (Sampath et al., 2014).

Deficits in attention are likely contributors to the core pathophysiology of autism spectrum disorder (ASD). Sustained, spatially divided attention in a prenatal valproic acid (VPA) model of ASD using the 5 Choice Serial Reaction Time Task (5-CSRTT) was examined. It was found that both male and female VPA rats had progressively lower accuracy and higher omissions with increasing attentional demands during 5-CSRTT training, and showed further performance decrements when subjected to parametric task manipulations. An important finding was that in a fixed rule setting, VPA rats could meet performance criteria when provided with a graded, extended training regime (Anshu et al., 2017).

\section{Pain and Stress}

VPCI, Delhi have demonstrated illustrating role of nitric oxide $(\mathrm{NO})$ in regulating stress effects and which 
may act as a therapeutic target for development of novel strategies against stress related disorders (Gulati et al., 2015). Dehydroepiandrosterone sulphate, ADMA and estrogen protective effect was illustrated in restraint stress (RS) induced alteration in neurobehavioural and brain oxidative injury in rats (Chakraborti et al., 2014). Recently, Pal et al. (2016) evaluated the protective effects of melatonin in RSinduced behavioural and immunological changes in rats. Thakur et al. (2017) investigated the effects of chronic predictable stress (CPS) and chronic unpredictable stress (CUS) on immunological responses in KLH immunized rats and involvement of NOergic signaling pathways mediating such responses.

NIPER-Ahmedabad is trying to dissect out the cellular and molecular mechanisms involved in chronic neuropathic pain and associated CNS co-morbidities. They aim to develop the novel therapeutics for the treatment of spontaneous ongoing pain devoid of drug addiction.

NIPER-Hyderabad has been working on physiology and pharmacotherapy of neuropathic pain associated with diabetes, chemotherapy and trauma. Research focuses on the pathogenic assessment and treatment of peripheral neuropathies in animal and cell culture models. They are working on various aspects involved in pharmacological evaluation of phytochemicals and small molecule modulators of signaling proteins in neuropathic pain models such as diabetic neuropathy, chemotherapy induced neuropathy, chronic constriction injury (CCI) of sciatic nerve. They have observed that such neuronal damage is persistent due to maladaptive activation of PARP enzyme and associated neuroinflammation and bioenergetic depletion in peripheral nerves (Komirishetty et al., 2016a). They have also observed that PARP inhibitors and phytochemical antioxidants administration to CCI operated rats reverse this neurological injury and associated neuropathic pain by controlling the expression of PARP enzyme to a physiologically minimal level (Komirishetty et al., 2016b; 2017). In addition to PARP (Poly ADP Ribose Polymerase), they have also studied the therapeutic benefit of dual regulation of Nrf2 and NF- $3 \mathrm{~B}$ pathways in CCI induced neuropathy (Arruri et al., 2017). The institute is also working on chemotherapy induced peripheral neuropathy, a dose limiting side effect associated with several chemotherapeutic agents. They have observed that oxaliplatin exposure leads to accumulation of damaged mitochondria leading to enhanced ROS generation and severe nerve damage contributing to pain under experimental conditions. Phytochemical modulators ameliorated mitochondrial deficits leading to reduced ROS burden and enhanced the intraepidermal nerve fibre density in oxaliplatin treated rats (Areti et al., 2017a; 2017b). They had elaborated the studies in experimental diabetic neuropathy, where they have started working on the role of mitochondrial dysfunction in the pathogenesis of diabetes associated neuronal dysfunction. In particular, the lab is involved in exploring the therapeutic benefits of promoting mitochondrial biogenesis and autophagy by adenosine monophosphate activated protein kinase (AMPK) and sirtuin deacetylase (SIRT1) modulation (Yerra et al., 2017a; 2017b).

At AIIMS, New Delhi, role of adenosinergic system, particularly the non specific antagonist theophylline in pain and possible mechanisms have been investigated (Katyal and Gupta, 2012). Recently Katyal et al. have demonstrated an analgesic activity of S-adenosylmethionine (SAM) and reversal of tolerance to analgesic effect of morphine with SAM using tail flick test (Katyal et al., 2017). This work has implications for drug addiction as well.

\section{Encephalitis}

Based upon pre-clinical study undertaken at National Brain Research Center (NBRC), Manesar, Haryana, a Phase III clinical trial has been completed at King George's Medical University, Lucknow, where minocycline has been used as a therapy for the patients with Japanese Encephalitis (JE) and Acute Encephalitis Syndrome (AES) (Kumar et al., 2016). It was observed in this trial that minocycline has some beneficial effect in patients especially in those patients who survived the initial days in hospital. Recently, another publication from Baba Raghav Das Medical College, Gorakhpur showed the therapeutic efficacy of minocycline in JE patients. Together these findings indicated that minocycline had a potential to be used commercially for JE and AES patients. Moreover, based upon findings from both the studies it may be possible to include minocycline in the management of AES and JE. At present NBRC and Department of 
Biotechnology is closely working with Director General Health Services, National Vector Borne Diseases Control Program and ICMR to consider minocycline for AES/JE patients in a pilot project. Recently, ICMR and Health ministry announced that minocycline would be used for an observational study for JE and AES patients.

\section{Neurotoxicity}

NIPER-Ahmedabad illustrated that microglial cells are the primary threat sensors of CNS which become activated in many pathological conditions. Using rat primary microglial cultures, dichlorvos exposure activated and induced apoptotic cell death in microglia (Sunkaria et al., 2012). Moreover, the effect of dichlorvos on crucial cell cycle regulatory proteins and the DNA damage sensor ataxia-telangiectasia mutated was also assessed. These findings suggested that dichlorvos can induce oxidative as well as nitrosative stress in microglia, which might be responsible for observed DNA damage, mitotic arrest, and ultimately apoptotic cell death in these cells (Sunkaria et al., 2013). So, it was hypothesized that if the production of oxidative/nitrosative stressors is restricted, the microglial over-activation can be prevented, and hence its downstream effects. To achieve this, a potent NO scavenger and SOD (superoxide dismutase) mimetic, 4-hydroxy TEMPO (4-HT) was investigated. DNA fragmentation analysis showed significant reduction in the DNA damage of 4-HT pre-treated microglia as compared to dichlorvos treated cells (Sunkaria et al., 2014a).

Next, the effect of dichlorvos mediated microglial activation on neuronal cells was studied. TDCM-treated neurons showed significant decrease in the expression of these pro-apoptotic molecules (Sunkaria et al., 2014b). Taken together, these findings suggest that 4-HT can significantly attenuate dichlorvos-induced microglial activation and prevent apoptotic neuronal cell death.

At University College of Medical Sciences (UCMS), Delhi neurotoxicity due to exposure to pesticide is being targeted. Melatonin and 4'chlorodiazepam significantly reversed the propoxurinduced cognitive impairment and oxidative stress parameters (Mehta et al., 2010; 2014).

\section{Miscellaneous}

Banaras Hindu University, Varanasi, has worked extensively on mitochondrial energetic with special reference to neurodegenerative diseases. Mitochondrial bioenergetics was characterized after neurodegenerative diseases in spinal cord injurymodel and neonatal anoxic model of rats (Samaiya et al., 2015). Neonatal anoxia led to time dependent progression of mitochondrial linked apoptosis in rat cortex and associated long term sensorimotor deficits (Samaiya et al., 2016; 2017). Silibinin ameliorated lipopolysaccharide-(LPS) induced memory deficits in experimental animals (Joshi et al., 2014). Piracetam attenuated LPS-induced neuroinflammation and cognitive impairment in rats (Tripathi et al., 2017). The discovery of 3 hydroxy 3 phenacyloxindole analogues of Isatin was found to have monoamine oxidase inhibitory activity (Tripathi et al., 2016). Further, the synthesis and pharmacological evaluation of N3-aryl/heteroaryl-substituted 2-(2-chlorostyryl)6, 7-dimethoxy-quinazolin-4 (3H)-ones illustrated anticonvulsant activity (Das et al., 2014b).

Abrupt discontinuation of chronic ethanol consumption produces severe withdrawal symptoms. Mirtazapine was found to be having better potential therapeutic use and profile over clonidine in overcoming alcohol withdrawal associated symptoms (Arora and Vohora, 2016).

At UIPS, UGC-CAS, Panjab University, Chandigarh, protective effect of curcumin, resveratrol against chronic alcohol-induced cognitive dysfunction in adult rats was mediated through anti-oxidant and anti-inflammatory mechanisms (Tiwari and Chopra, 2013a; 2013b). Curcumin and tocotrienol treatment prevented cognitive deficits in rat pups postnatally exposed to ethanol involving NF-к $\beta$ mediated apoptotic signaling simulating children with fetal alcohol spectrum disorders (Tiwari and Chopra, 2012; Tiwari et al., 2012).

\section{Conclusion}

It can be concluded from above studies that neuropharmacology research clinically and preclinically has flourished but there is an urgent need to appraise the level of research, number of publications and patents for an international standing. A greater focus on enhancing infrastructure and funding will be beneficial. 


\section{Acknowledgements}

The authors would like to thank all esteemed colleagues and laboratories for their co-operation in compiling this manuscript.

\section{References}

Ahmad A, Rasheed N, Gupta P, Singh S, Siripurapu KB, Ashraf GM, Kumar R, Chand K, Maurya R, Banu N, Al-Sheeha $M$ and Palit G (2012) Novel Ocimumoside A and B as anti-stress agents: modulation of brain monoamines and antioxidant systems in chronic unpredictable stress model in rats Phytomedicine 19 639-647

Anshu K, Nair A K, Kumaresan U D, Kutty B M, Srinath S and Laxmi T R (2017) Altered attentional processing in male and female rats in a prenatal valproic acid exposure model of ASD Autism Res doi: 10.1002/aur.1852

Anwar MJ, Radhakrishna K V, Sharma A and Vohora D (2014) Raloxifene preserves phenytoin and sodium valproate induced bone loss by modulating serum estradiol and TGF$\beta 3$ content in bone of female mice Eur J Pharm Sci 62219 226

Areti A, Komirishetty P, Akuthota M, Malik R A and Kumar A (2017a) Melatonin prevents mitochondrial dysfunction and promotes neuroprotection by inducing autophagy during oxaliplatin evoked peripheral neuropathy J Pineal Res 62 doi: 10.1111/jpi.12393

Areti A, Komirishetty P and Kumar A (2017b) Carvedilol prevents functional deficits in peripheral nerve mitochondria of rats with oxaliplatin-evoked painful peripheral neuropathy Toxicol Appl Pharmacol 322 97103

Arora S and Vohora D (2016) Comparative evaluation of partial $\alpha_{2}$-adrenoceptor agonist and pure $\alpha_{2}$-adrenoceptor antagonist on the behavioural symptoms of withdrawal after chronic alcohol administration in mice Basic Clin Pharmacol Toxicol 119 202-209

Arora T, Bhowmik M, Khanam R and Vohora D (2013) Oxcarbazepine and fluoxetine protect against mouse models of obsessive compulsive disorder through modulation of cortical serotonin and creb pathway Behav Brain Res 247 146-152

Arruri V, Komirishetty P, Areti A, Dungavath S K N and Kumar A (2017) Nrf2 and NF-êB modulation by Plumbagin attenuates functional, behavioural and biochemical deficits in rat model of neuropathic pain Pharmacol Reports 69 $625-632$

Atchaneeyasakul K, Guada L, Ramdas K, Watanabe M,
Bhattacharya P, Raval AP and Yavagal DR (2016) Large Animal Canine Endovascular Ischemic Stroke Models: A Review Brain Res Bull 127 134-140

ATTEND Collaborative Group (2017) Family-led rehabilitation after stroke in India (ATTEND): a randomised controlled trial Lancet 390 588-599

Banerjee T K and Das S K (2016) Fifty years of stroke researches in India Ann Indian Acad Neurol 19 1-8

Bhowmik M, Khanam R and Vohora D (2015) Activation of AKT/GSK3â pathway by TDZD-8 attenuates kainic acid induced neurodegeneration but not seizures in mice Neurotoxicology 46 44-52

Bhowmik M, Saini N and Vohora D (2014) Histamine H3 antagonism by ABT-239 attenuates kainic acid induced excitotoxicity in mice Brain Res 1581 129-140

Biswas J, Goswami P, Gupta S, Joshi N, Nath C and Singh S (2016) Streptozotocin Induced Neurotoxicity Involves Alzheimer's Related Pathological Markers: A study on N2A Cells Mol Neurobiol 53 2794-2806

Biswas J, Gupta S, Verma D K and Singh S (2017) Streptozotocin alters glucose transport, connexin expression and endoplasmic reticulum functions in neurons and astrocytes Neuroscience 356 151-166

Chakraborti A, Gulati K and Ray A (2014) Possible role of nitric oxide (NO) in the regulation of gender related differences in stress induced anxiogenesis in rats Nitric Oxide $\mathbf{4 3} 74$ 80

Chatterjee M, Singh S, Kumari R, Verma A K and Palit G (2012) Evaluation of the antipsychotic potential of Panaxquinquefolium in ketamine induced experimental psychosis model in mice Neurochem Res 3 7759-770

Chatterjee M, Verma R, Kumari R, Singh S, Verma A K, Dwivedi A K and Palit G (2015) Antipsychotic activity of standardized Bacopa extract against ketamine-induced experimental psychosis in mice: Evidence for the involvement of dopaminergic, serotonergic, and cholinergic systems Pharm Biol 53 1850-1860

Chatterjee M, Verma R, Lakshmi V, Sengupta S, Verma AK, Mahdi AA and Palit G (2013) Anxiolytic effects of Plumeria rubra var. acutifolia (Poiret) L. flower extracts in the elevated plus-maze model of anxiety in mice Asian $J$ Psychiatr 6 113-118

Chaudhary N, Kabra M, Gulati S, Gupta Y K, Pandey R M and Bhatia B D (2016) Frequencies of CYP2C9 polymorphisms in North Indian population and their association with drug levels in children on phenytoin monotherapy BMC Pediatr 66 doi 10.1186/s12887-0160603-0 
Chauhan A, Sharma U, Reeta K H, Jagannathan N R, Mehra R D and Gupta Y K(2012) Neuroimaging, biochemical and cellular evidence of protection by mycophenolatemofetil on middle cerebral artery occlusion induced injury in rats Eur J Pharmacol 684 71-78

Das N R, Gangwal R P, Damre M V, Sangamwar A T and Sharma S S (2014a) A PPAR- $\beta / \delta$ agonist is neuroprotective and decreases cognitive impairment in a rodent model of Parkinson's disease Curr Neurovasc Res 11114-124

Das N, Garabadu D, Banerjee A G, Krishnamurthy S and Shrivastava S K (2014b) Synthesis and pharmacological evaluation of some N3-aryl/heteroaryl-substituted 2-(2chlorostyryl)-6, 7-dimethoxy-quinazolin-4 (3H)-ones as potential anticonvulsant agents Med Chem Res 23 41674176

Devi M G, Sharma A, Mohanty S, Jain N, Verma K, Padma M V, Pal P, Chabbra H S, Khadilkar S, Prabhakar S and Singh G (2016) Report: Stem cell applications in neurological practice, an expert group consensus appraisal Ann Indian Acad Neurol 19367-373

Devi P U, Saraogi P, Manocha A and Vohora D (2012) Pharmacological and biochemical analysis of interactions between $\mathrm{N}$-acetylcysteine and some antiepileptic drugs on experimental seizures in mice CNS Neurosci Ther 18 406-413

Devinsky O, Hesdorffer D C, Thurman D J, Lhatoo S and Richerson G (2016) Sudden unexpected death in epilepsy: epidemiology, mechanisms, and prevention Lancet Neurol 15 1075-1088

Dogra S, Kumar A, Umrao D, Sahasrabuddhe A A and Yadav P N (2016) Chronic Kappa opioid receptor activation modulates NR2B: Implication in treatment resistant depression Sci Rep 633401

Dubey H, Gulati K and Ray A (2017a) Recent studies on cellular and molecular mechanisms in Alzheimer's disease: Focus on epigenetic factors and histone deacetylase Reviews in the Neurosciences (In Press)

Dubey H, Gulati K and Ray A (2017b) Effects of Nitric Oxide (NO) Modulators on Cognitive Function and Brain Oxidative Stress in Experimental Model of Alzheimer's Disease in Rats J Pharmacol Rep 2126

Dwivedi R, Gupta Y K, Singh M, Joshi R, Tiwari P, Kaleekal T and Tripathi M (2015)Correlation of saliva and serum free valproic acid concentrations in persons with epilepsy Seizure 25 187-190

Dwivedi R, Singh M, Kaleekal T, Gupta Y K and Tripathi M (2016) Concentration of antiepileptic drugs in persons with epilepsy: A comparative study in serum and saliva
Int J Neurosci $126972-978$

Dwivedi S, Nagarajan R, Hanif K, Siddiqui HH, Nath C and Shukla R (2013) Standardized Extract of Bacopa monniera Attenuates Okadaic Acid Induced Memory Dysfunction in Rats: Effect on Nrf2 Pathway Evid Based Complement Alternat Med 2013294501

Fatima S, Haque R, Jadiya P, Shamsuzzama, Kumar L and Nazir A (2014) Ida-1, the Caenorhabditiselegans orthologue of mammalian diabetes autoantigen IA-2, potentially acts as a common modulator between Parkinson's disease and Diabetes: role of Daf-2/Daf-16 insulin like signaling pathway PLoS One 9e113986

Garabadu D and Krishnamurthy S (2014a) Asparagus racemosus attenuates anxiety-like behavior in experimental animal models Cell Mol Neurobiol 34 511-521

Garabadu D and Krishnamurthy S (2014b) Diazepam potentiates the antidiabetic, antistress and anxiolytic activities of metformin in type-2 diabetes mellitus with cooccurring stress in experimental animals BioMed Res Int 2014693074

Garabadu D, Ahmad A and Krishnamurthy S (2015) Risperidone Attenuates Modified Stress-Re-stress Paradigm-Induced Mitochondrial Dysfunction and Apoptosis in Rats Exhibiting Post-traumatic Stress Disorder-Like Symspace ptoms J Mol Neurosci 56 299-312

Garabadu D, Reddy B H and Krishnamurthy S (2015a) Citalopram protects against cold-restraint stress-induced activation of brain-derived neurotrophic factor and expression of nuclear factor kappa-light-chain-enhancer of activated B cells in rats $J$ Mol Neurosci 55 355-66

Garabadu D, Shah A, Singh S and Krishnamurthy S (2015b) Protective effect of eugenol against restraint stress-induced gastrointestinal dysfunction: Potential use in irritable bowel syndrome Pharm boil 53 968-974

Gaur V and Kumar A (2012) Effect of nonselective and selective COX-2 inhibitors on memory dysfunction, glutathione system, and tumor necrosis factor alpha level against cerebral ischemia reperfusion injury Drug Chem Toxicol 35 218-224

Geed M, Garabadu D, Ahmad A and Krishnamurthy S (2014) Silibinin pretreatment attenuates biochemical and behavioral changes induced by intrastriatal MPP+ injection in rats Pharmacol Biochem Behav 117 92-103

Goswami P, Gupta S, Biswas J, Joshi N, Swarnkar S, Nath C and Singh S (2016a) Endoplasmic Reticulum Stress Plays a Key Role in Rotenone-Induced Apoptotic Death of Neurons Mol Neurobiol 53 285-298

Goswami P, Gupta S, Biswas J, Sharma S and Singh S (2016b) Endoplasmic Reticulum Stress Instigates the Rotenone 
Induced Oxidative Apoptotic Neuronal Death: a Study in Rat Brain Mol Neurobiol 53 5384-5400

Gourie-Devi M (2014) Epidemiology of neurological disorders in India: Review of background, prevalence and incidence of epilepsy, stroke, Parkinson's disease and tremors Neurol India 62 588-598

Gulati K and Ray A (2014) Differential neuromodulatory role of NO in anxiety and seizures: an experimental study Nitric Oxide 43 55-61

Gulati K, Joshi J C and Ray A (2015) Recent advances in stress research: focus on nitric oxide Eur J Pharmacol 765 406414

Gupta S and Gupta Y K (2017) Combination of Zizyphus jujuba and silymarin showed better neuroprotective effect as compared to single agent in MCAo-induced focal cerebral ischemia in rats $J$ Ethnopharmacol 197 118-127

Gupta S, Goswami P, Biswas J, Joshi N, Sharma S, Nath C and Singh S (2015) 6-hydroxydopamine and lipopolysaccharides induced DNA damage in astrocytes: involvement of nitric oxide and mitochondria Mutat Res Genet Toxicol Environmen Mutagen 778 22-36

Gupta S, Sharma U, Jagannathan N R and Gupta Y K (2017) Neuroprotective effect of lercanidipine in middle cerebral artery occlusion model of stroke in rats Exp Neurol $\mathbf{2 8 8}$ 25-37

Haque R and Nazir A (2016a) Identification and functional characterization of a putative IDE, C28F5.4 (ceIDE-1), in Caenorhabditiselegans: Implications for Alzheimer's disease Biochim Biophys Acta 1860 2454-2462

Haque R and Nazir A (2016b) SMAD Transcription Factor, Sma-9, Attunes TGF- $\beta$ Signaling Cascade towards Modulating Amyloid Beta Aggregation and Associated Outcome in Transgenic C. elegans Mol Neurobiol 53 109119

Husain I, Akhtar M, Abdin M Z, Islamuddin M, Shaharyar M and Najmi A K (2017a) Rosuvastatin ameliorates cognitive impairment in rats fed with high-salt and cholesterol diet via inhibiting acetylcholinesterase activity and amyloid beta peptide aggregation Hum Exp Toxicol 1 960327117705431

Husain I, Akhtar M, Vohora D, Abdin M Z, Islamuddin M, Akhtar M J and Najmi A K (2017b) Rosuvastatin Attenuates High-Salt and Cholesterol Diet Induced Neuroinflammation and Cognitive Impairment via Preventing Nuclear Factor KappaB Pathway Neurochem Res 42 2404-2416

Jadhav V, Bhattacharya P and Yavagal D (2015) Intra-arterial approaches to stem cell therapy for stroke Cell Therapy
Springer Verlag, USA ISBN:978-3-319-15062-8

Jadiya P and Nazir A (2014) A Pre- and Co-Knockdown of RNAseT Enzyme, Eri-1, Enhances the Efficiency of RNAi Induced Gene Silencing in Caenorhabditis elegans PLoS One 9 e 87635

Jadiya P, Mir S S and Nazir A (2012) Effect of various classes of pesticides on expression of stress genes in transgenic $C$. elegans model of Parkinson's disease CNS Neurol Disord Drug Targets 11 1001-1005

Jain P, Shastri S, Gulati S, Kaleekal T, Kabra M, Gupta N, Gupta Y K and Pandey R M (2015) Prevalence of UGT1A6 polymorphisms in children with epilepsy on valproate monotherapy Neurol India 63 35-39

Joshi R, Garabadu D, Teja G R and Krishnamurthy S (2014) Silibinin ameliorates LPS-induced memory deficits in experimental animals Neurobiol Learn Mem 116117-131

Joshi R, Reeta K H, Sharma S K, Tripathi M and Gupta Y K (2015a) Pharmacodynamic and pharmacokinetic interaction of PanchagavyaGhrita with phenytoin and carbamazepine in maximal electroshock induced seizures in rats Ауи 36 196-202

Joshi R, Reeta K H, Sharma S K, Tripathi M and Gupta Y K (2015b) PanchagavyaGhrita, an Ayurvedic formulation attenuates seizures, cognitive impairment and oxidative stress in pentylenetetrazole induced seizures in rats Indian J Exp Biol 53 446-451

Joshi R, Tripathi M, Gupta P, Gulati S and Gupta Y K (2017) Adverse effects \& drug load of antiepileptic drugs in patients with epilepsy: Monotherapy versus polytherapy Indian J Med Res 145 317-326

Kamat P K and Nath C (2015) Okadaic acid: a tool to study regulatory mechanisms for neurodegeneration and regeneration in Alzheimer's disease Neural Regen Res 10 365-367

Kamat P K, Tota S K, Rai S, Shukla R, Ali S, Najmi A K and Nath C (2012) Okadaic acid induced neurotoxicity leads to central cholinergic dysfunction in rats Eur J Pharmacol 690 90-98

Kamat PK, Tota S K, Rai S, Shukla R, Ali S, Najmi A K and Nath C (2013) Okadaic acid (ICV) induced tau phosphorylation: a role of NMDA receptor Neuroscience 238 97-113

Kamat P K, Rai S, Swarnkar S, Shukla R and Nath C (2014) Mechanism of synapse redox stress in Okadaic acid (ICV) induced memory impairment: Role of NMDA receptor Neurochem Int 76 32-41

Katyal J and Gupta Y K (2012) Dopamine release is involved in antinociceptive effect of theophylline Int J Neurosci 122 $17-21$ 
Katyal J, Kumar H and Gupta Y K (2015) Anticonvulsant activity of the cyclooxygenase-2 (COX-2) inhibitor etoricoxib in pentylenetetrazole-kindled rats is associated with memory impairment Epilepsy_Behav 44 98-103

Katyal J, Kumar H, Joshi D and Gupta Y K (2017) S-adenosyl methionine (SAM) attenuates the development of tolerance to analgesic activity of morphine in rats Neurosci Lett 645 $67-73$

Katyal J, Sarangal V and Gupta Y K (2012) Interaction of hydroalcoholic extract of Acoruscalamus Linn. with sodium valproate and carbamazepine Indian J Exp Biol 50 51-55

Komirishetty P, Areti A, Gogoi R, Sistla R and Kumar A (2017) Combination strategy of PARP inhibitor with antioxidant prevent bioenergetic deficits and inflammatory changes in CCI-induced neuropathy Neuropharmacology 113 137147

Komirishetty P, Areti A, Sistla R and Kumar A (2016b) Morin mitigates chronic constriction injury (CCI)-induced peripheral neuropathy by inhibiting oxidative stress induced PARP over-activation and neuroinflammation Neurochem Res 41 2029-2042

Komirishetty P, Areti A, Yerra V G, Ruby P, Sharma S S, Gogoi R, Sistla R and Kumar A (2016a) PARP inhibition attenuates neuroinflammation and oxidative stress in chronic constriction injury induced peripheral neuropathy Life Sci 150 50-60

Krishnamurthy S, Garabadu D and Ranga R N (2013a) Asparagus racemosus modulates the hypothalamic-pituitary-adrenal axis and brain monoaminergic systems in rats Nutr Neurosci 16 255-261

Krishnamurthy S, Garabadu D and Joy K P (2013b) Risperidone ameliorates post-traumatic stress disorder-like symptoms in modified stress re-stress model Neuropharmacology 75 $62-77$

Kumar B, Modi M, Saikia B and Medhi B (2017a) Evaluation of Brain Pharmacokinetic and Neuropharmacodynamic Attributes of an Antiepileptic Drug, Lacosamide, in Hepatic and Renal Impairment: Preclinical Evidence ACS Chem Neurosci 8 1589-1597

Kumar H, Katyal J and Gupta Y K (2015) Low dose zinc supplementation beneficially affects seizure development in experimental seizure models in rats Biol Trace Elem Res 163 208-216

Kumar R, Basu A, Sinha S, Das M, Tripathi P, Jain A, Kumar C, Atam V, Khan S and Singh A S (2016) Role of oral Minocycline in acute encephalitis syndrome in India - a randomized controlled trial BMC Infect Dis 1667
Kumar S, Mishra A and Krishnamurthy S (2017b) Purinergic Antagonism Prevents Mitochondrial Dysfunction and Behavioral Deficits Associated with Dopaminergic Toxicity Induced by 6-OHDA in Rats Neurochem Res 24 1-7

Megiddo I, Colson A, Chisholm D, Dua T, Nandi A and Laxminarayan R (2016) Health and economic benefits of public financing of epilepsy treatment in India: An agentbased simulation model Epilepsia 57 464-474

Mehla J, Pahuja M, Dethe S M, Agarwal A and Gupta YK (2012) Amelioration of intracerebroventricular streptozotocin induced cognitive impairment by Evolvulus alsinoides in rats: in vitro and in vivo evidence Neurochem Int 6110521064

Mehla J, Pahuja M, Gupta P, Dethe S, Agarwal A and Gupta Y K (2013a) Clitoria ternatea ameliorated the intracerebroventricularly injected streptozotocin induced cognitive impairment in rats: Behavioral and biochemical evidence Psychopharmacology (Berl) 230 589-605

Mehla J, Pahuja M and Gupta Y K (2013b) Streptozotocininduced sporadic Alzheimer's disease: selection of appropriate dose $J$ Alzheimers Dis 33 17-21

Mehta A K, Halder S, Khanna N, Tandon O P, Singh U R and Sharma K K (2012) Role of NMDA and opioid receptors in neuropathic pain induced by chronic constriction injury of sciatic nerve in rats J Basic Clin Physiol Pharmacol 23 49-55

Mehta K D, Mehta A K, Halder S, Khanna N, Tripathi A K and Sharma K K (2014) Protective effect of melatonin on propoxur-induced impairment of memory and oxidative stress in rats Environ Toxicol 29 705-713

Misra S, Chopra K, Saikia U N, Sinha V R, Sehgal R, Modi M and Medhi B (2016) Effect of mesenchymal stem cells and galantamine nanoparticles in rat model of Alzheimer's disease Regen Med 11 629-646

Nasir S A, Sharma A, Khanam R and Vohora D (2012) Effect of medroxyprogesterone on development of PTZ-induced kindling in mice Neuroscience 207 283-287

Niranjan R, Nagarajan R, Hanif K, Nath C and Shukla R (2014) LPS induces mediators of neuroinflammation, cell proliferation, and GFAP expression in human astrocytoma cells U373MG: the anti-inflammatory and anti-proliferative effect of guggulipid Neurol Sci 35 409-414

Padma Srivastava MV (2016) Management of stroke: The triumphs and the travails Neurol India 64 (S1) 6-7

Pahuja M, Kaleekal T, Reeta K H, Tripathi M and Gupta Y K (2012a) Interaction profile of Zizyphus jujuba with phenytoin, phenobarbitone, and carbamazepine in maximal 
electroshock-induced seizures in rats Epilepsy Behav $\mathbf{2 5}$ 368-373

Pahuja M, Mehla J, Reeta K H, Joshi S and Gupta Y K (2012b) Root extract of Anacyclus pyrethrum ameliorates seizures, seizure-induced oxidative stress and cognitive impairment in experimental animals Epilepsy_Res 98 157-165

Pahuja M, Mehla J, Reeta KH, Tripathi M and Gupta Y K (2013) Effect of Anacyclus pyrethrum on pentylenetetrazoleinduced kindling, spatial memory, oxidative stress and rhokinase II expression in mice Neurochem Res 38 547-556

Pal R, Gulati K, Banerjee B D and Ray A (2016) Pharmacological and biochemical studies on the protective effects of melatonin during stress-induced behavioral and immunological changes in relation to oxidative stress in rats Can J Physiol Pharmacol 94 296-301

Pottoo F H, Bhowmik M and Vohora D (2014) Raloxifene protects against seizures and neurodegeneration in a mouse model mimicking epilepsy in postmenopausal woman Eur $J$ Pharm Sci 65 167-173

Prajapati K D, Sharma S S and Roy N (2012) Hepatocyte nuclear factor-1alpha mediated upregulation of albumin expression in focal ischemic rat brain Neurol Res 34 25-31

Prajapati S K, Garabadu D and Krishnamurthy S (2017) Coenzyme Q10 Prevents Mitochondrial Dysfunction and Facilitates Pharmacological Activity of Atorvastatin in 6OHDA Induced Dopaminergic Toxicity in Rats Neurotox Res 31 478-492

Prakash A, Medhi B and Chopra K (2013a) Granulocyte colony stimulating factor (GCSF) alone and in combination with memantine improves not only memory but also co-morbid associated Alzheimer's \& Dementia: The J Alzheimer's Association $9 \mathrm{P} 499$

Prakash A, Medhi B and Chopra K (2013b) Granulocyte colony stimulating factor (GCSF) improves memory and neurobehavior in an amyloid-â induced experimental model of Alzheimer's disease Pharmacol Biochem Behav 110 4657

Rai S, Kamat P K, Nath C and Shukla R (2014) Glial activation and post-synaptic neurotoxicity: the key events in Streptozotocin (ICV) induced memory impairment in rats Pharmacol Biochem Behav 117 104-117

Rajput S K, Sharma A K, Meena C L, Pant A B, Jain R and Sharma S S (2016) Effect of L-pGlu-(1-benzyl)-1-His-1-Pro-NH2 against in-vitro and in-vivo models of cerebral ischemia and associated neurological disorders Biomed Pharmacother 84 1256-1265

Rashid D, Panda B P and Vohora D (2015) Reduced estradiol synthesis by letrozole, an aromatase inhibitor, is protective against development of pentylenetetrazole-induced kindling in mice Neurochem Int 90 271-274

Reeta K H, Prabhakar P and Gupta Y K (2016) Anticonvulsant activity of the antidepressant drug, tianeptine, against pentylenetetrazole-induced seizures mitigates cognitive impairment in rats Behav Pharmacol 27 623-632

Reeta K H, Singh D and Gupta Y K (2017a) Chronic treatment with taurine after intracerebroventricular streptozotocin injection improves cognitive dysfunction in rats by modulating oxidative stress, cholinergic functions and neuroinflammation Neurochem Int 108 146-156

ReetaKH, Singh D and Gupta Y K (2017b) Edaravone attenuates intra- cerebroventricular streptozotocin-induced cognitive impairment in rats Eur J Neurosci 45 987-997

Sahu J K, Gulati S, Kabra M, Arya R, Sharma R, Gupta N, Kaleekal T, Reeta K H and Gupta Y K (2012) Evaluation of subclinical hypothyroidism in ambulatory children with controlled epilepsy on valproate monotherapy $J$ Child Neurol 27 594-597

Samaiya P K and Krishnamurthy S (2015) Characterization of mitochondrial bioenergetics in neonatal anoxic model of rats J Bioenerg Biomembr 47 217-222

Samaiya P K, Narayan G, Kumar A and Krishnamurthy S (2016) Neonatal anoxia leads to time dependent progression of mitochondrial linked apoptosis in rat cortex and associated long term sensorimotor deficits Int J Dev Neurosci 52 5565

Samaiya P K, Narayan G, Kumar A and Krishnamurthy S (2017) Tempol (4 hydroxy-tempo) inhibits anoxia-induced progression of mitochondrial dysfunction and associated neurobehavioral impairment in neonatal rats $J$ Neurol Sci 375 58-67

Sampath D, Sabitha K R, Hegde P, Jayakrishnan H R, Kutty B M, Chattarji S, Rangarajan G and Laxmi T R (2014) A study on fear memory retrieval and REM sleep in maternal separation and isolation stressed rats Behav Brain Res 273 144-154

Sarangi S C, Joshi D, Kumar R, Kaleekal T and Gupta Y K (2017) Pharmacokinetic and pharmacodynamic interaction of hydroalcoholic extract of Ocimum sanctum with valproate Epilepsy Behav 75 203-209

Sarangi S C, Tripathi M, Kakkar A K and Gupta Y K (2014) Effect of antiepileptic therapy on trace elements status in Indian population in a tertiary care hospital from northern India: a cross sectional study Epilepsy Res 108 917-927

Sarangi S C, Tripathi M, Kakkar A K and Gupta Y K (2016) Comparison of body composition in persons with epilepsy on conventional $\&$ new antiepileptic drugs 
Indian J Med Res 143 323-330

Sarmah D, Saraf J, Kaur H, Pravalika K, Tekade R K, Borah A, Kalia K, Dave K R and Bhattacharya P (2017) Stroke Management: An Emerging Role of Nanotechnology Micromachines 8 1-13

Sewal R K, Modi M, Saikia U N, Chakrabarti A and Medhi B (2017) Increase in seizure susceptibility in sepsis like condition explained by spiking cytokines and altered adhesion molecules level with impaired blood brain barrier integrity in experimental model of rats treated with lipopolysaccharides Epilepsy Res 135 176-186

Sharma S, Padma M V, Bhardwaj A, Sharma A, Sawal N and Thakur S (2016) Telestroke in resource-poor developing country model Neurol India 64 934-940

Sharma S, Sethi G R and Gupta S (2017) Standard Treatment Guidelines: A Manual for Medical Therapeutics Publisher Delhi Society for Promotion of Rational Use of Drugs and Wolters Kluwer Health, Delhi, $5^{\text {th }}$ edition (under print)

Shinomol G K, Bharath M M and Muralidhara (2012b) Pretreatment with Bacopa monnieri extract offsets 3nitropropionic acid induced mitochondrial oxidative stress and dysfunctions in the striatum of prepubertal mouse brain Can J Physiol Pharmacol 90 595-606

Shinomol G K, Mythri R B, Srinivas Bharath M M and Muralidhara (2012a) Bacopa monnieri extract offsets rotenone-induced cytotoxicity in dopaminergic cells and oxidative impairments in mice brain Cell Mol Neurobiol 32 455-465

Singh R K, Diwan M, Dastidar S G and Najmi A K (2017a) Differential effect of p38 and MK2 kinase inhibitors on the inflammatory and toxicity biomarkers in vitro Hum Exp Toxicol 960327117715901

Singh R K, Najmi A K and Dastidar S (2017b) Biological functions and role of mitogen-activated protein kinase activated protein kinase 2 (MK2) in inflammatory diseases Pharmacol Reports 69 746-756

Singh S, Mishra A and Shukla S (2016) ALCAR Exerts Neuroprotective and Pro-Neurogenic Effects by Inhibition of Glial Activation and Oxidative Stress via Activation of the Wnt/3-Catenin Signaling in Parkinsonian Rats $\mathrm{Mol}$ Neurobiol 53 4286-42301

Singh S, Mishra A and Shukla S (2017a) ALCAR promote adult hippocampal neurogenesis by regulating cell-survival and cell death-related signals in rat model of Parkinson's disease like-phenotypes Neurochem Int 108 388-396

Singh S, Mishra A, Srivastava N and Shukla S (2017b) MK-801 (Dizocilpine) Regulates Multiple Steps of Adult Hippocampal Neurogenesis and Alters Psychological
Symptoms via Wnt/â-Catenin Signaling in Parkinsonian Rats ACS Chem Neurosci 8 592-605

Srinivasan K and Sharma S S (2012a) 3-Bromo-7-nitroindazole attenuates brain ischemic injury in diabetic stroke via inhibition of endoplasmic reticulum stress pathway involving CHOP Life Sci 90 154-160

Srinivasan K and Sharma S S (2012b) Neuroprotection Offered by Edaravone in a Diabetic Stroke Model is mediating through Inhibition of Endoplasmic Reticulum Basic Clin Pharmacol Toxicol 110 133-140

Sunkaria A, Sharma D R, Wani W Y and Gill K D (2014a) 4Hydroxy TEMPO attenuates dichlorvos induced microglial activation and apoptosis ACS Chem Neurosci 5 115-127

Sunkaria A, Sharma D R, Wani W Y and Gill K D (2014b) Attenuation of dichlorvos-induced microglial activation and neuronal apoptosis by 4-hydroxy TEMPO Mol Neurobiol 49 163-75

Sunkaria A, Wani W Y, Sharma D R and Gill K D (2012) Dichlorvos exposure results in activation induced apoptotic cell death in primary rat microglia Chem Res Toxicol 25 $1762-1770$

Sunkaria A, Wani W Y, Sharma D R and Gill K D (2013) Dichlorvos-induced cell cycle arrest and DNA damage repair activation in primary rat microglial cells $J$ Neurosci Res 91 444-452

Surapaneni D K, Adapa S R, Preeti K, Teja G R, Veeraragavan M and Krishnamurthy S (2012) Shilajit attenuates behavioral symptoms of chronic fatigue syndrome by modulating the hypothalamic-pituitary-adrenal axis and mitochondrial bioenergetics in rats $J$ Ethnopharmacol 143 91-99

Thakur A K, Chatterjee S S and Kumar V (2013) Beneficial effects of Brassica juncea on cognitive functions in rats Pharm Biol 51 1304-1310

Thakur A K, Chatterjee S S and Kumar V (2014a) Antidepressantlike effects of Brassica juncea L. leaves in diabetic rodents Indian J Exp Biol 52 613-622

Thakur A K, Chatterjee S S and Kumar V (2014b) Neuropsychopharmacology of a therapeutically used Andrographis paniculata extract: a preclinical study Orient Pharm Exp Med 14 181-191

Thakur A K, Chatterjee S S and Kumar V (2014c) Adaptogenic potential of andrographolide: An active principle of the king of bitters (Andrographis paniculata) J Tradit Complement Med 5 42-50

Thakur A K, Rai G, Chatterjee S S and Kumar V (2016) Beneficial effects of an Andrographis paniculata extract and andrographolide on cognitive functions in streptozotocininduced diabetic rats PharmBiol 54 1528-1538 
Thakur A K, Soni U K, Rai G, Chatterjee S S and Kumar V (2014d) Protective effects of Andrographis paniculata extract and pure andrographolide against chronic stresstriggered pathologies in rats Cell Mol Neurobiol 341111 1121

Thakur T, Gulati K, Rai N and Ray A(2017) Experimental studies on possible regulatory role of nitric oxide on the differential effects of chronic predictable and unpredictable stress on adaptive immune responses Internat Immunopharmacol 50 236-242

Thomas J, Khanam R and Vohora D (2016) Augmentation of antidepressant effects of venlafaxine by agomelatine in mice are independent of kynurenine pathway Neurochemistry International 99 103-09

Tiwari V, Arora V and Chopra K (2012) Attenuation of NF- $\kappa \beta$ mediated apoptotic signaling by tocotrienol ameliorates cognitive deficits in rats postnatally exposed to ethanol Neurochem Int 61 310-320

Tiwari V and Chopra K (2012) Attenuation of oxidative stress, neuroinflammation, and apoptosis by curcumin prevents cognitive deficits in rats postnatally exposed to ethanol Psychopharmacology (Berl) 224 519-535

Tiwari V and Chopra K (2013a) Protective effect of curcumin against chronic alcohol-induced cognitive deficits and neuroinflammation in the adult rat brain Neuroscience $\mathbf{2 4 4}$ 147-158

Tiwari V and Chopra K (2013b) Resveratrol abrogates alcoholinduced cognitive deficits by attenuating oxidativenitrosative stress and inflammatory cascade in the adult rat brain Neurochem Int 62 861-869

Tota S, Goel R, Pachauri S D, N R, Najmi A K, Hanif K and Nath C (2013a) Effect of angiotensin II on spatial memory, cerebral blood flow, cholinergic neurotransmission, and brain derived neurotrophic factor in rats Psychopharmacology (Berl) 226 357-369

Tota S, Hanif K, Kamat P K, Najmi A K and Nath C (2013b) Role of central angiotensin receptors in scopolamine-induced impairment in memory, cerebral blood flow, and cholinergic function Psychopharmacology (Berl) 226 357-369

Tota S, Kamat P K, Saxena G, Hanif K, Najmi A K and Nath C (2012b) Central angiotensin converting enzyme facilitates memory impairment in intracerebroventricular streptozotocin treated rats Behav Brain Res 226 317-330

Tota S, Nath C, Najmi A K, Shukla R and Hanif K (2012a) Inhibition of central angiotensin converting enzyme ameliorates scopolamine induced memory impairment in mice: Role of cholinergic neurotransmission, cerebral blood flow and brain energy metabolism Behav Brain Res 232 66-76

Tripathi A, Paliwal P and Krishnamurthy S (2017) Piracetam Attenuates LPS-Induced Neuroinflammation and Cognitive Impairment in Rats Cell Mol Neurobiol doi: 10.1007/ s10571-017-0468-2

Tripathi R K, Krishnamurthy S and Ayyannan S R (2016) Discovery of 3 Hydroxy 3 phenacyloxindole Analogues of Isatin as Potential Monoamine Oxidase Inhibitors Chem Med Chem 11 119-132

Uppalapati D, Das N R, Gangwal R P, Damre M V, Sangamwar A T and Sharma S S (2014) Neuroprotective Potential of Peroxisome Proliferator Activated Receptor-5ØüPAgonist in Cognitive Impairment in Parkinson's Disease: Behavioral, Biochemical, and PBPK Profile PPAR Research: 2014, doi:10.1155/2014/753587

Verma D K, Joshi N, Raju K S, Wahajuddin M, Singh R K and Singh S (2015)_Metabolic enhancer piracetam attenuates rotenone induced oxidative stress: a study in different rat brain regions Acta Neurobiol Exp (Wars) 75 399-411

World Health Organisation Neurological Disorders: Public Health Challenges Geneva:World Health Organisation; 2006 http:/ /www.who.int/mental_health/neurology/neurodiso/en/

Yerra V G and Kumar A (2017a) Adenosine monophosphateactivated protein kinase abates hyperglycaemia-induced neuronal injury in experimental models of diabetic neuropathy: effects on mitochondrial biogenesis, autophagy and neuroinflammation Mol Neurobiol 54 23012312

Yerra V G, Kalvala A K and Kumar A (2017b) Isoliquiritigenin reduces oxidative damage and alleviates mitochondrial impairment by SIRT1 activation in experimental diabetic neuropathy $J$ NutrBiochem 47 41-52. 